\title{
Empirical Treatment of Highly Suspected Nontuberculous Mycobacteria Infections Following Aesthetic Procedures
}

\author{
Hyung Rok Kim, Eul Sik Yoon, Deok Woo Kim, Na Hyun Hwang, Yoo Seok Shon, \\ Byung Il Lee, Seung-Ha Park \\ Department of Plastic Surgery and Reconstructive Surgery, Korea University Medical Center, Korea University College of Medicine, Seoul, \\ Korea
}

Background Infection caused by nontuberculous mycobacteria (NTM) has been increasing. Awareness of this infection is crucial yet problematic. Delayed management may lead to destructive results. We empirically treated a series of patients with clinical suspicion of NTM infection prior to the identification of the pathogen.

Methods A total of 12 patients who developed surgical site infections between January 2011 and February 2014 were reviewed. Patients with a skin and subcutaneous infection resistant to standard management over two weeks, and previous history of aesthetic procedures within three months were regarded as highly suspected of having an NTM infection. A variety of diagnostic modalities were examined simultaneously, along with starting empirical treatment including a combination of clarithromycin and moxifloxacin, and surgical debridement.

Results All wounds healed completely within 4 weeks. The mean follow-up duration was 7.2 months, and none of the patients developed relapse. Specific NTM pathogens were identified in six patients. Eight patients showed caseating granuloma implying an NTM infection. One patient showed an uncommon Stenotrophomonas infection, which was successfully treated. Three patients had no evidence of a pathogen despite repeated microbial tests. Complications such as scarring, pigmentation, and disfigurement were common in all the patients.

Conclusions NTM should be considered in the differential diagnosis of an unusual skin and soft-tissue infection. We propose an empirical regimen of clarithromycin and moxifloxacin as an efficient treatment option for an NTM infection.

Keywords Mycobacterium infections, nontuberculous / Soft-tissue infections / Early diagnosis / Antibiotics, antitubercular
Correspondence: Eul-Sik Yoon Department of Plastic Surgery and Reconstructive Surgery, Korea University Medical Center, Korea University College of Medicine, 73 Inchon-ro, Seongbuk-gu, Seoul 136-705, Korea

Tel: +82-2-920-5368

Fax: +82-2-922-7437

E-mail:yesanam2@korea.ac.kr

This article was presented at the 12th Korea-Japan Congress of Plastic and Reconstructive Surgery on May 15, 2014, in Incheon, Korea.

No potential conflict of interest relevant to this article was reported.

Received: 9 Jun 2014 • Revised: 10 Jul 2014 • Accepted: 17 Jul 2014

pISSN: 2234-6163 • elSSN: 2234-6171 • http://dx.doi.org/10.5999/aps.2014.41.6.759 • Arch Plast Surg 2014;41:759-767

\section{INTRODUCTION}

Recently, nontuberculous mycobacteria (NTM), often called mycobacteria other than tuberculosis (MOTT), have attracted increasing attention, as they have been causing infectious diseases in the healthy population. According to Runyon's classification, NTM can be classified into rapidly growing mycobacteria (RGM) and slow-growing mycobacteria (SGM), on the basis of 
growth rates and pigmentation patterns [1]. Among the four groups of NTM species, group IV consists of the fast growers, which can produce a positive culture within 7 days [2]. These RGM have recently been found to be a common pathogen of skin and soft-tissue infections that develop after aesthetic procedures such as liposuction, fat grafts, filler injection, and implant insertion [3]. Many clinicians have attempted the use of various diagnostic methods to confirm a suspected NTM pathogen, but the confirmation takes too much time, and hence, appropriate treatments cannot be begun on time. Moreover, misdiagnosis and the delay of applying appropriate antibiotics obscure the treatment process, which results in patients suffering severe aesthetic sequelae, such as scarring, pigmentation, and disfigurement. The purpose of this study is to define "highly suspected NTM infection" patients as those with suspicious clinical manifestations and a history of previous aesthetic procedures and to evaluate the outcome of the empirical treatment prior to the confirmation of the pathogens.

\section{METHODS}

\section{Study group}

A total of 83 patients who developed postoperative skin and soft-tissue infections between January 2011 and February 2014 were retrospectively reviewed. Twelve patients who had more than three criteria applicable, including one major criterion, were defined as the "highly suspected NTM infection" group. The specific inclusion criteria are described in Table 1. The other 71 patients were excluded from this study.

\section{Diagnostic modalities}

All the 12 patients defined as "highly suspected NTM infection" patients experienced surgical debridement in the operating room under local anesthesia their first visit. A sufficient volume of tissue specimens was harvested in the debridement procedure in order to simultaneously conduct the following six labo-

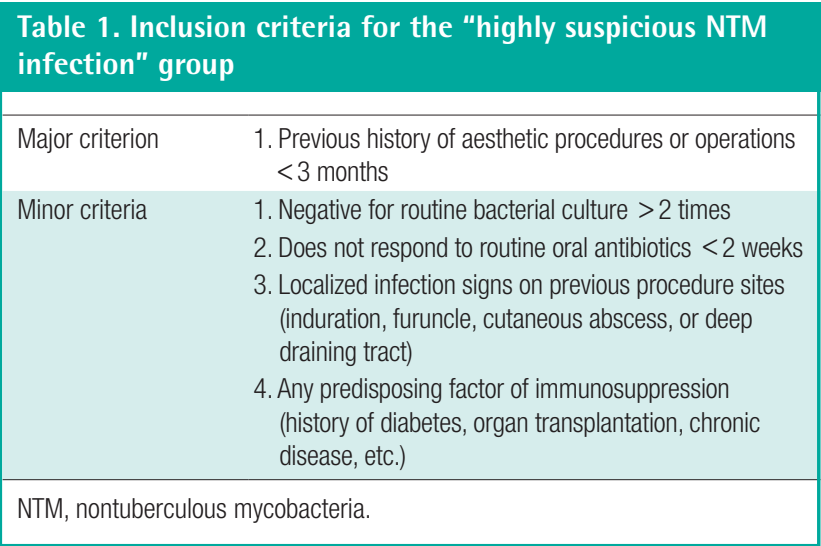

ratory tests to confirm the pathogen: 1) Gram stain, 2) bacterial tissue culture, 3) acid-fast bacilli (AFB) stain, 4) mycobacterial culture, 5) real-time polymerase chain reaction (PCR) for $M y$ cobacterium tuberculosis and NTM species, and 6) microscopic histopathology (Fig. 1).

\section{Treatment}

All patients were treated with surgical debridement along with combination antibiotics (clarithromycin and moxifloxacin). Surgical debridement was conducted on the first visit for all patients, and empirical antibiotics were administered on the same day as that of surgical debridement. Nine patients presenting localized furuncle, abscesses, deep draining sinus tracts, or indolent seroma were treated with a combination of oral antibiotics (clarithromycin $500 \mathrm{mg} \mathrm{q} 12$ hours+moxifloxacin $400 \mathrm{mg} \mathrm{q} 24$ hours) and daily dressing at the outpatient clinics. The other three patients showing systemic inflammatory signs such as fever, chilling, sweating, and lymphadenopathy were treated with a combination of intravenous antibiotics and repeated debridement in the operating room during their hospital stays. This combination antibiotic therapy was discontinued only when 1) a positive bacterial tissue culture was confirmed and the wound was successfully cured with susceptible antibiotics, and 2) the culture for mycobacteria was confirmed to be negative after 6 weeks of incubation. The overall treatment strategy is summarized in Fig. 2.

\section{RESULTS}

The patients consisted of 11 women and 1 man, ranging in age from 22 to 52 years, with a median age of 38 years. All the patients were initially misdiagnosed with pyogenic injection abscess and treated with repeated curettage and oral antibiotics at local clinics. None of the patients had positive microbial labora-

\section{Fig. 1. Simultaneous laboratory tests for pathogen detection}

We executed surgical debridement on the day of the patient's first visit in order to wash out the wound efficiently and to obtain sufficient tissue specimens to conduct six different laboratory tests. AFB, acid-fast bacteria; TB, tuberculosis; PCR, polymerase chain reaction.

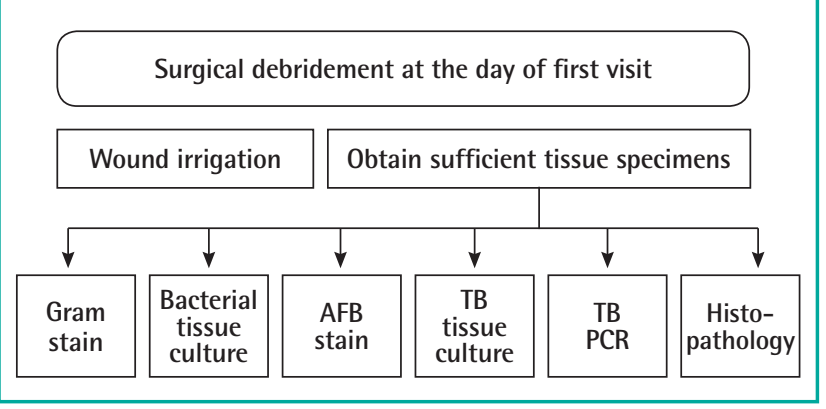




\section{Fig. 2. Flowchart of empirical treatment}

Flowchart of empirical treatment for highly suspected nontuberculous mycobacteria infection. AFB, acid-fast bacteria; TB, tuberculosis; NTM, nontuberculous mycobacteria; PCR, polymerase chain reaction; MIC, minimal inhibitory concentration.

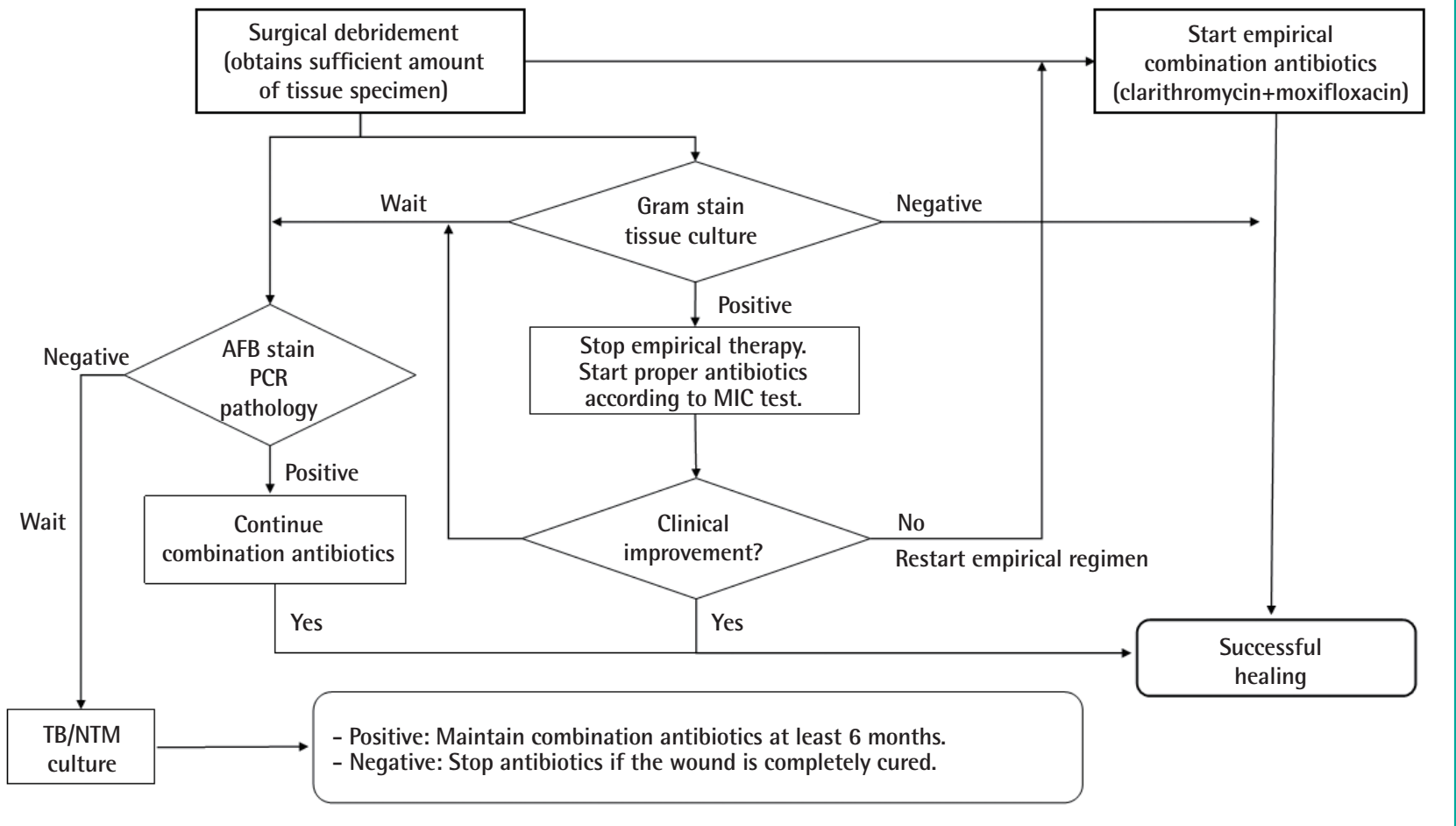

\section{Table 2. Demographic and clinical data of 12 patients with chronic inflammation followed by aesthetic procedures}

\begin{tabular}{|c|c|c|c|c|c|c|c|c|c|}
\hline Case & $\begin{array}{l}\text { Sex/Age } \\
\text { (yr) }\end{array}$ & Site & Previous procedure & Cutaneous symptoms & $\begin{array}{l}\text { Gram } \\
\text { staining }\end{array}$ & $\begin{array}{c}\text { AFB } \\
\text { staining }\end{array}$ & Culture & $\begin{array}{l}\text { TB/NTM } \\
\text { PCR }\end{array}$ & Histopathology \\
\hline 1 & $F / 39$ & Lt. cheek & Fat graft & Erythema, abscess & $\mathrm{N}$ & $\mathrm{N}$ & M. abscessus & NA & $\begin{array}{l}\text { Chronic caseating } \\
\text { granulomatous } \\
\text { inflammation }\end{array}$ \\
\hline 2 & $F / 37$ & Rt. breast & $\begin{array}{l}\text { Breast augmentation } \\
\text { with silicone implant, } \\
\text { fat graft }\end{array}$ & $\begin{array}{l}\text { Erythema, induration, } \\
\text { abscess }\end{array}$ & N & $P$ & M. abscessus & $P$ & $\begin{array}{l}\text { Chronic caseating } \\
\text { granulomatous } \\
\text { inflammation }\end{array}$ \\
\hline 3 & $\mathrm{~F} / 50$ & Lt. cheek & Fat graft & Erythema, induration & N & N & N & N & $\begin{array}{l}\text { Caseating granulomatous } \\
\text { inflammation }\end{array}$ \\
\hline 4 & $\mathrm{~F} / 30$ & Lt. thigh & Liposuction & Erythema, induration & N & N & M. chelonae & $P$ & $\begin{array}{l}\text { Chronic caseating } \\
\text { granulomatous } \\
\text { inflammation }\end{array}$ \\
\hline 5 & $\mathrm{~F} / 52$ & Glabella & Filler injection & Erythema, abscess & N & N & $\mathrm{N}$ & NA & $\begin{array}{l}\text { Acute and chronic } \\
\text { inflammation }\end{array}$ \\
\hline 6 & $\mathrm{~F} / 40$ & Nasal dorsum & Rhinoplasty & Erythema & $\mathrm{N}$ & N & N & NA & NA \\
\hline 7 & $M / 29$ & Scalp & $\begin{array}{l}\text { Ommaya reservoir } \\
\text { insertion }\end{array}$ & Abscess, sinus tract & $\mathrm{N}$ & N & N & $\mathrm{N}$ & Chronic inflammation \\
\hline 8 & $F / 22$ & Chin & Primary repair & Induration & $\mathrm{N}$ & N & N & N & $\begin{array}{l}\text { Caseating granulomatous } \\
\text { inflammation }\end{array}$ \\
\hline 9 & $F / 22$ & Forehead & Fat graft & $\begin{array}{l}\text { Abscess, sinus tract, } \\
\text { skin necrosis }\end{array}$ & $\mathrm{N}$ & $P$ & M. fortuitum & $P$ & $\begin{array}{l}\text { Chronic caseating } \\
\text { granulomatous } \\
\text { inflammation }\end{array}$ \\
\hline 10 & $\mathrm{~F} / 41$ & Rt. temple area & Fat graft & $\begin{array}{l}\text { Erythema, abscess, } \\
\text { skin necrosis }\end{array}$ & $\mathrm{N}$ & N & $\mathrm{N}$ & N & $\begin{array}{l}\text { Chronic caseating } \\
\text { granulomatous } \\
\text { inflammation }\end{array}$ \\
\hline 11 & $F / 42$ & Rt. cheek & Fat graft & Erythema, abscess & N & N & S. maltophilia & N & $\begin{array}{l}\text { Acute inflammation with } \\
\text { abscess formation }\end{array}$ \\
\hline 12 & $F / 31$ & Rt. breast & Fat graft & Erythema, depression & N & N & M. fortuitum & $P$ & $\begin{array}{l}\text { Caseating granulomatous } \\
\text { inflammation }\end{array}$ \\
\hline
\end{tabular}

TB, tuberculosis; NTM, nontuberculous mycobacteria; PCR, polymerase chain reaction; F, female; Lt., left; Rt., right; N, negative; NA, not available; P, positive; M, male. 
tory results showing either bacterial or fungal species at the time of visit. Demographic data, clinical manifestations, and results of various diagnostic modalities were retrospectively reviewed and are summarized in Table 2.

The mean follow-up duration was 7.2 months, and none of the patients developed any recurrence. Specific NTM pathogens were identified in six patients. The histopathology results of eight patients showed a caseating granulomatous inflammation strongly implying an NTM infection. A patient showed an uncommon bacterial infection (Stenotrophomonas maltophilia), which was susceptible to intravenous moxifloxacin therapy. Three patients had no evidence of pathogens despite repeated microbial tests. Complications at the site of infection, including hypertrophic scarring, pigmentation, and disfigurement, were common in all the patients.

\section{Case 1}

A 22-year-old female patient who had undergone autologous fat grafting for forehead augmentation, presented with partial skin necrosis, erythematous nodules, and multiple microabscesses on the forehead (Fig. 3A). She showed systemic infection signs, such as fever $\left(>38.3^{\circ} \mathrm{C}\right)$, chills, and cervical lymphadenopathy. Initial incision and drainage was performed at a local clinic, but it was not effective. She presented to our hospital two weeks after the lesions had aggravated. Regular microbial tests such as Gram staining and culture were all negative. AFB staining was positive, and the histopathology result of a chronic caseating granulomatous inflammation was strong evidence of an NTM infection. Mycobacterium fortuitum was confirmed after 6 weeks of incubation.

During the 3 weeks of hospitalization, clarithromycin (500 $\mathrm{mg}$, q 12 hours) and moxifloxacin ( $400 \mathrm{mg} /$ day) were given intravenously. Daily irrigation and curettage were also performed, and the symptoms improved within 3 weeks. After being discharged, the patient was prescribed with oral clarithromycin (500 mg, q 12 hours) and moxifloxacin ( $400 \mathrm{mg} /$ day) for 6 months. She was followed-up for an additional 2 months after stopping medication (Fig. 3B).

\section{Case 2}

A 31-year-old female who had undergone autologous fat grafting, presented with erythematous indurations and microabscesses on the medial side of her right breast (Fig. 4A). Incision, drainage, and antibiotic treatment were initiated at the local clinic, but the lesions did not see any improvements for 2 weeks. Gram staining and culture were negative. As a mycobacterial infection was strongly suspected, AFB staining and cultures for $M$. tuberculosis and NTM were performed. While AFB staining was negative, $M$. fortuitum was identified by an NTM culture.

On the first visit, the patient was treated with empirical use of clarithromycin (500 mg, q 12 hours) and moxifloxacin (400 $\mathrm{mg} /$ day) along with surgical debridement. One additional debridement was necessary, and her symptoms improved within 3 weeks. After the confirmation of the M. fortuitum infection, the patient was maintained on the medication for 6 months. She was followed-up for an additional 8 months without any relapse (Fig. 4B).

\section{Fig. 3. Case 1}

A 22-year-old female patient who developed multiple pustules and partial skin necrosis on the forehead after autologous fat grafting. She was diagnosed with a Mycobacterium fortuitum infection. (A) Before treatment. (B) Four months after treatment.
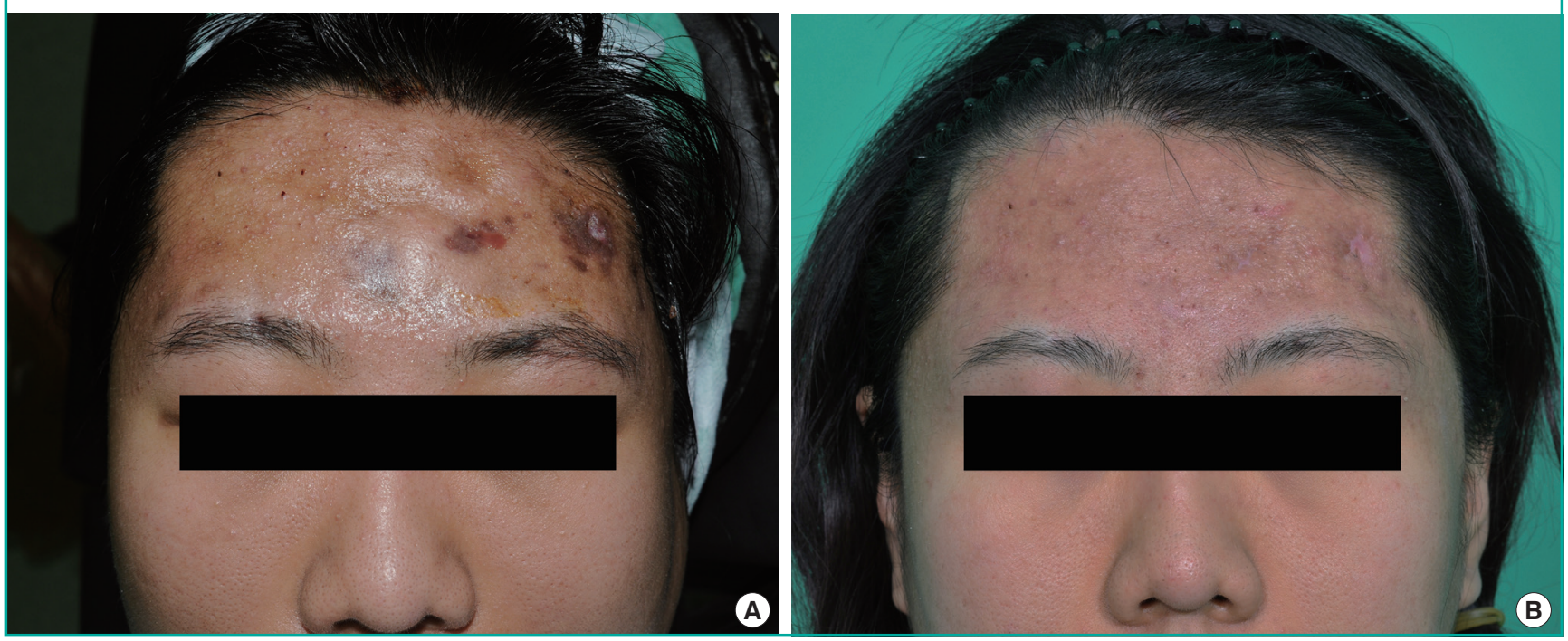


\section{Case 3}

A 50-year-old woman went through autologous fat grafting and presented multiple erythematous nodules on the left cheek (Fig. $5 \mathrm{~A})$. One month after the procedure, lesions developed on the left side of her face. She visited our hospital one month after lesion development. Gram staining and culture were negative. Staining for AFB and cultures for $M$. tuberculosis and NTM were performed along with a microscopic study. The AFB staining and the culture test were negative. Despite a series of negative results, the histopathology result showed a caseating granulomatous inflammation, which initially led to the assumption that this case was an NTM infection. Incision, drainage, and curettage were performed on the day of the clinic visit, and two additional surgical debridement procedures followed. As a result, the patient's symptoms improved within 2 weeks. The patient was given empirical antibiotics of oral clarithromycin $(500 \mathrm{mg}$, q 12 hours) and moxifloxacin ( $400 \mathrm{mg} /$ day) for six weeks since the first day of visit, and she stopped medication after the negative result of the NTM culture was confirmed. She was followed-up for 8 months without relapse; however, a depressed, contracted scar remained (Fig. 5B).

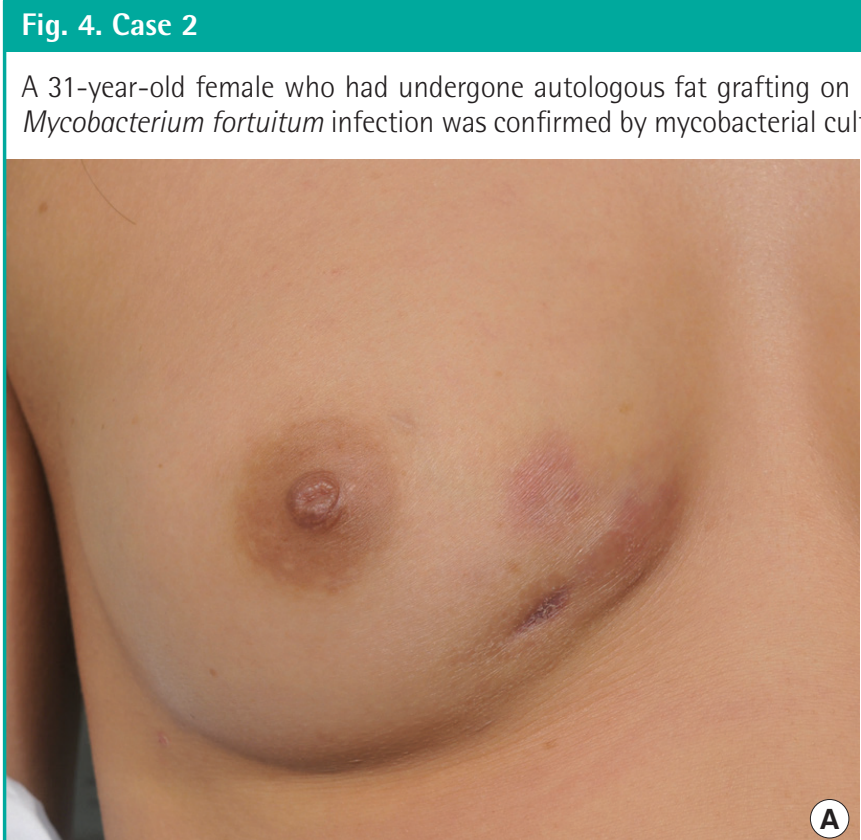

Fig. 5. Case 3

A 50-year-old woman who underwent autologous fat grafting on her cheek showed erythematous nodules and purulent discharge. (A) Before treatment. (B) Six months after treatment.
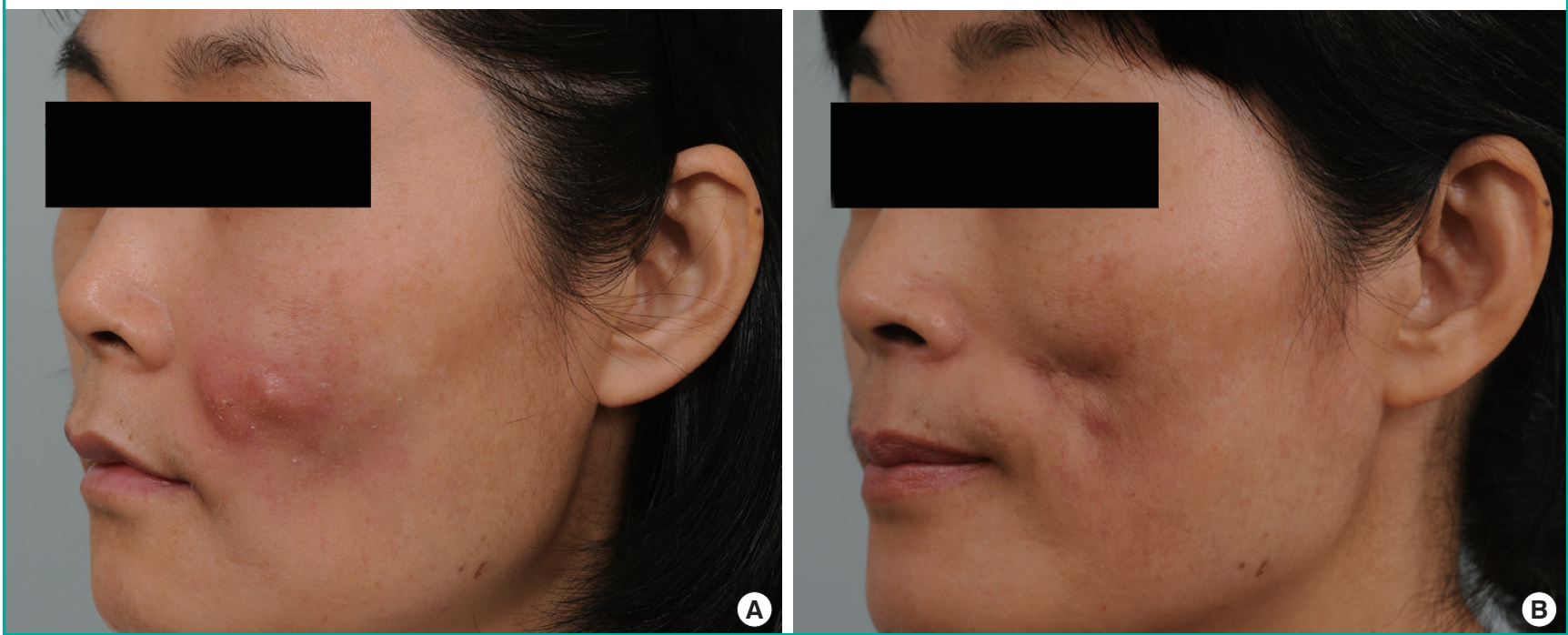


\section{DISCUSSION}

Infections caused by the RGM species are relatively uncommon. However, several studies have reported that outbreaks of a nosocomial surgical site RGM infection, most often due to $M y$ cobacterium abscessus or $M$. fortuitum, have been associated with augmentation mammoplasty, liposuction, and facial plastic surgery $[2,4,5]$. Clinical diagnosis of mycobacterial skin and softtissue infections is not easy to perform, and even when performed correctly, the diagnosis often takes a significant amount of time for the final confirmation. In fact, there have been instances in which delays of more than a year have been reported [6]. Some of the key reasons for the difficulty of a speedy and accurate diagnosis are 1) high costs for the polymerase chain reaction test in order to detect NTM, 2) a relatively long time of 6-8 weeks for the mycobacterial culture incubation period, and 3) a lack of awareness of the possibility of NTM infections. Surgeons should recognize the possibility when caring for patients with chronic skin and soft-tissue infections following an invasive aesthetic procedure.

A series of case reports of a total of 88 patients of NTM softtissue infections had been widely reviewed in order to reveal common clinical history and disease courses among the patients. All patients in the literature had experiences of invasive procedures such as acupuncture, fat grafts, liposuction, and implant insertion. Interestingly, 70 patients $(79.5 \%)$ suffering from uncontrolled infections were reported to visit a hospital within three months (range, 10 days to 12 weeks) after their first exposure $[3,7-10]$. On the basis of the experiences reported in the literature, we formulated the clinical criteria consisting of one major and four minor factors that could discriminate the patients suspected of NTM infections from those with pyogenic infections. We assumed the history of invasive procedures within three months to be the key factor in our criteria. Even though a few cases reported the detection of a pathogen more than three months after the initial exposure, we determined three months to be a reasonable period to cover a majority of patients with NTM infections. The minor criteria reflected clinical symptoms, laboratory findings, and comorbidities that were commonly found among patients with NTM infections.

There have been numerous clinical and laboratory trials for the early confirmation of mycobacterial infections. Initially, we focused on patients infected surgically with atypical cutaneous manifestations localized at the sites of previous aesthetic procedures. According to our findings, skin and soft-tissue infections of NTM look slightly different from pyogenic bacterial infections. First, the color of erythema around the focal lesion is darker and more purplish than the typical reddish color of pyo- genic induration. This is probably due to the fact that the natural course of an NTM infection is chronic, and the pathogen continually induces hyperpigmentation of the affected skin lesions [11]. Second, NTM cutaneous infections seem more likely to form deep nodules with multiple deep sinus tracts containing fluids that seem to be more serous than the sticky yellowish pus observed in the case of bacterial infections [12]. These were not critical features but played important roles in the identification of NTM infections.

The diagnosis of NTM can be also made on the basis of histopathological findings. The presence of central caseous necrosis with amorphous granular debris, loss of cellular detail, and the presence of Langhan's giant cells are sufficient characteristics to make a reasonably accurate diagnosis of a mycobacterial infection. Although a number of different diseases such as brucellosis, mycoses, actinomycosis, and syphilis are involved in the formation of granuloma, mycobacterial infection is the most common microbial cause of caseating granuloma [13]. In the case of the histologic results that showed caseating granuloma, we considered these findings to be an NTM infection until the mycobacterial culture result was confirmed to be negative. The positive findings of the histopathological exam allowed us to make an earlier start of empirical treatments for NTM.

Universally, the AFB stain and culture have been widely used in the diagnosis of mycobacterial infections. Even though the AFB stain is known to be a rapid, convenient, and cost-effective method, it has low sensitivity, ranging from $22 \%$ to $78 \%$, and is unable to discriminate NTM from M. tuberculosis [14]. Culture, on the other hand, demonstrates a higher sensitivity than an AFB stain, as well as nearly $100 \%$ specificity in the diagnosis of mycobacterial infections; however, this method requires an extended time of $6-8$ weeks.

Recent advancement in mycobacteriology laboratory techniques plays a critical role in the laboratory diagnosis of tuberculosis and of diseases caused by NTM. Advanced PCR tests are now widely used in the early detection of NTM infections and species identification. These techniques include restriction fragment length polymorphism (RFLP), PCR-reverse blot hybridization assay (REBA), and real-time PCR. RFLP uses restriction enzymes to break DNA into fragments, which are then compared with one another to distinguish the difference in the DNA sequences. Although this technique is convenient, many variables can affect results because of the use of electrophoresis in the comparison of the DNA fragments [15]. PCR-REBA utilizes an oligonucleotide probe that specifically binds to certain DNA sites of specific pathogens, enabling a rapid identification of the 19 most common mycobacterial species, including M. abscessus, M. fortuitum complex, and Mycobacterium chelonae 
[2]. Both the sensitivity of NTM detection and the specificity are $100 \%$ for the DNA from the cultured isolated specimens [16]. The success rates for NTM identification obtained by targeting the $16 \mathrm{~S}$ rRNA, rpoB, and $h s p 65$ genes are $65 \%, 82 \%$, and $87 \%$, respectively [17].

In this study, we used an AdvanSure TB/NTM real-time PCR kit (LG Life Sciences, Seoul, Korea) for the detection of mycobacteria. Real-time PCR both detects and quantifies the DNA as the reaction progresses. Despite its limitation in terms of the number of pathogens that can be identified in a single exam, this technique has high specificity and sensitivity, and allows a quantitative analysis of the pathogen. The AdvanSure kit collects the products from two simultaneous reactions: one reaction occurs between the probe and the rpoB gene site of NTM or the IS6110 site of the $M$. tuberculosis complex, and the other reaction occurs when the TaqMan probe is broken down by hybridization. Due to the simultaneous use of primer and probe hybridization, AdvanSure has a sensitivity and a specificity of $100 \%$. Other studies pertaining to sensitivity and specificity in relation to this product have reported a sensitivity of $92.7 \%$ and a specificity of $91.3 \%$ [18]. In our institution, the cost of a real-time PCR test is eight times the cost of an AFB smear. However, as real-time PCR has the advantages of a higher detection rate and a shorter result waiting time of 2.3 days on average, we strongly recommended this test to all the patients. Despite our efforts, 3 out of 12 patients refused the PCR test because of its high cost.

Throughout the study, surgical incision, drainage, and debridement were executed in the operating room at the patients' first visit. When performing a wide excision or drainage of abscesses under local anesthesia, more than $3 \mathrm{~mL}$ of the tissue specimen was collected in most patients in order to conduct various laboratory studies simultaneously. This was done under the assumption that a sufficient amount of tissue specimen will decrease the rate of false negative results. Moreover, infection wounds were washed with saline far more thoroughly and efficiently in the operating room.

In earlier studies on the NTM treatment, empirical cephalosporin or ampicillin antibiotics were usually changed to antiNTM medication including macrolides when obtaining laboratory evidence such as positive AFB staining or a positive PCR test $[2,19]$. Throughout this study, the focus was on the inclusion criteria of patients "highly suspected of NTM infection," which emphasized the previous history of aesthetic procedures, atypical cutaneous manifestations, and serial negative microbial tests. Thus, an empirical regimen of clarithromycin and moxifloxacin (Orally or IV) targeting the NTM infection was initiated from the day of the first visit without hesitation despite the absence of the NTM infection evidence at that time. As a result, the start date of empirical antibiotics could be pushed ahead by 2-3 days on the basis of these decisions.

Treatment guidelines published jointly by the American Thoracic Society and the Infectious Diseases Society of America (ATS/IDSA) are a valuable resource for the practicing clinician [1]. These guidelines focus on initial management and emphasize that susceptibility testing should be performed on all mycobacterial isolates. It is known that an effective treatment of NTM skin infections includes surgical debridement along with a combination of antibiotic therapy. An antibiotic combination must be administered for a sufficiently long period of time to ensure complete wound healing and no recurrence. In order to prevent drug resistance and clinical relapse, antimicrobial therapy should be a multidrug regimen with a macrolide base as recommended [1].

A combination of clarithromycin and moxifloxacin was selected for the empirical regimen decided on the basis of multiple consultations by the infectious disease expert at our institution. We administered the combination regimen to the patients while waiting for microbial evidence on whether there was a strong suspicion of an atypical mycobacterial infection. Clarithromycin emerged as the single best empiric choice for cutaneous NTM infections [20]. Yu et al. [19] reported that the rate of drug resistance to clarithromycin in the case of RGM was $12.5 \%$. Rifampin, amikacin, imipenem, ciprofloxacin, and cefoxitin have all been used to good effect, provided that the disease was not at an advanced stage. Moxifloxacin is one of the most recently marketed fluoroquinolone antimicrobials. It differs from ciprofloxacin in having much better in vitro activity against Grampositive aerobes while retaining potent activity against Gramnegative aerobes. It is also active against the species of atypical mycobacteria associated with dermatologic infections. Moxifloxacin penetrates well into the inflammatory blister fluid and muscle and subcutaneous adipose tissues, which makes it a reasonable option for the treatment of skin and soft-tissue infections [10]. Gillespie et al. [21] evaluated the activity of moxifloxacin in comparison with that of four other quinolones and isoniazid against clinical isolates of mycobacteria. It proved to be the most active of the quinolones tested against $M$. tuberculosis $\left(\mathrm{MIC}_{90} 0.25 \mathrm{mg} / \mathrm{L}\right)$, Mycobacterium avium-intracellulare $\left(\mathrm{MIC}_{90}\right.$ $1.0 \mathrm{mg} / \mathrm{L}$ ), Mycobacterium kansasii $\left(\mathrm{MIC}_{90} 0.06 \mathrm{mg} / \mathrm{L}\right.$ ), and $M$. fortuitum $\left(\mathrm{MIC}_{90} 1 \mathrm{mg} / \mathrm{L}\right)$. Interactions between moxifloxacin and macrolides against clinical isolates of M. abscessus and Mycobacterium massiliense were also studied. The addition of moxifloxacin to macrolide therapy showed a treatment benefit to $M$. massiliense in contrast to negative influences on the treatment outcome of the M. abscessus infection [22].

Since clarithromycin and moxifloxacin together have a wide range of antimicrobial activity, it is a reasonable choice among 
the wide range of empirical agents. We experienced a single case of an atypical S. maltophilia infection followed by autologous fat grafting by repeated tissue cultures. The positive culture result was recognized 11 days after the start of the empirical treatment, and the pathogen was proven to be susceptible to moxifloxacin. The patient showed rapid clinical improvement within 2 weeks and continued oral moxifloxacin for 4 weeks despite the wound being completely healed. The efficient antimicrobial activity of moxifloxacin against $S$. maltophilia was widely reported in the cases of meningitis and keratitis treatment [23,24]. Moreover, this regimen was effective in three patients who did not demonstrate any evidence of the pathogen. We assumed that the broad spectrum of clarithromycin and moxifloxacin activity covered certain pathogens that were not isolated or identified.

We continued to prescribe this regimen for 4 weeks to one patient having an S. maltophilia infection and three patients showing no microbial evidence. This regimen was administered to three patients demonstrating caseous necrosis in histopathologic exams for at least 6 weeks until the mycobacterial culture was confirmed to be negative. Five patients confirmed to have an NTM infection were administered this regimen for 6 months. Although moxifloxacin and clindamycin were not always the best choice of antibiotics according to the susceptibility results, we decided to continue this regimen for the following reasons: First, all the five cases already showed complete wound healing state at the time when the susceptibility results were reported. Second, both macrolides and quinolones were demonstrated to be effective along with the minimum inhibitory concentration required to inhibit the growth of $90 \%$ of organisms $\left(\mathrm{MIC}_{90}\right)$, of less than $0.5 \mathrm{mg} / \mathrm{L}$ against the pathogens in all the cases. Moreover, the infectious disease expert expected the benefit of maintaining this regimen to far surpass the risk of adverse effects caused by antibiotic changes. Eight patients with caseating necrosis or NTM confirmation were followed up by the infectious disease expert as well. Most cases demonstrated clinical improvement within 2 weeks, and wound healing within 4 weeks after the first visit. Recurrence and any adverse effects associated with antibiotics were not observed during the follow-up period. The optimal length of therapy is unknown but is likely to be shorter than that in the case of a pulmonary or disseminated disease; current recommendations by ATS are for 6-12 months [1].

The empirical treatment prior to pathogen identification was effective for the highly suspicious group of NTM patients in terms of reducing the time taken for clinical improvement. Moreover, a regimen of a combination of clarithromycin and moxifloxacin demonstrated excellent treatment outcomes of NTM, atypical bacteria, and local inflammation of unknown causes. Simultaneous execution of laboratory tests also saved time in iden- tifying the pathogen. This empirical treatment strategy was based on multiple clues from the history and clinical manifestations and provided an efficient option for the management of skin and soft-tissue infections as compared to the standard management.

\section{REFERENCES}

1. Griffith DE, Aksamit T, Brown-Elliott BA, et al. An official ATS/IDSA statement: diagnosis, treatment, and prevention of nontuberculous mycobacterial diseases. Am J Respir Crit Care Med 2007;175:367-416.

2. Lim JM, Kim JH, Yang HJ. Management of infections with rapidly growing mycobacteria after unexpected complications of skin and subcutaneous surgical procedures. Arch Plast Surg 2012;39:18-24.

3. Murillo J, Torres J, Bofill L, et al. Skin and wound infection by rapidly growing mycobacteria: an unexpected complication of liposuction and liposculpture. The Venezuelan Collaborative Infectious and Tropical Diseases Study Group. Arch Dermatol 2000;136:1347-52.

4. Clegg HW, Foster MT, Sanders WE Jr, et al. Infection due to organisms of the Mycobacterium fortuitum complex after augmentation mammaplasty: clinical and epidemiologic features. J Infect Dis 1983;147:427-33.

5. Meyers H, Brown-Elliott BA, Moore D, et al. An outbreak of Mycobacterium chelonae infection following liposuction. Clin Infect Dis 2002;34:1500-7.

6. Gravante G, Caruso R, Araco A, et al. Infections after plastic procedures: incidences, etiologies, risk factors, and antibiotic prophylaxis. Aesthetic Plast Surg 2008;32:243-51.

7. Hsiao CH, Tsai TF, Hsueh PR. Characteristics of skin and soft tissue infection caused by non-tuberculous mycobacteria in Taiwan. Int J Tuberc Lung Dis 2011;15:811-7.

8. Liao CH, Lai CC, Ding LW, et al. Skin and soft tissue infection caused by non-tuberculous mycobacteria. Int J Tuberc Lung Dis 2007;11:96-102.

9. Macadam SA, Mehling BM, Fanning A, et al. Nontuberculous mycobacterial breast implant infections. Plast Reconstr Surg 2007; 119:337-44.

10. Guay DR. Moxifloxacin in the treatment of skin and skin structure infections. Ther Clin Risk Manag 2006;2:417-34.

11. Callender VD, St Surin-Lord S, Davis EC, et al. Postinflammatory hyperpigmentation: etiologic and therapeutic considerations. Am J Clin Dermatol 2011;12:87-99.

12. Elston D. Nontuberculous mycobacterial skin infections: recognition and management. Am J Clin Dermatol 2009; 10:281-5.

13. Zumla A, James DG. Granulomatous infections: etiology 
and classification. Clin Infect Dis 1996;23:146-58.

14. Yeam YS, Jeong OY, Jang SJ, et al. Comparison of culture, Acid-Fast stain and polymerase chain reaction assay for detection of mycobacterium tuberculosis. Korean J Clin Pathol 1995; 15:594-603.

15. Bagyalakshmi R, Senthilvelan B, Therese KL, et al. Application of polymerase chain reaction (PCR) and PCR based restriction fragment length polymorphism for detection and identification of dermatophytes from dermatological specimens. Indian J Dermatol 2008;53:15-20.

16. Molecules and Diagnositcs Incorporation. REBA Myco-ID product manual. Wonju, KR: Molecules and Diagnositcs Incorporation; 2009.

17. Kim BJ, Lee SH, Lyu MA, et al. Identification of mycobacterial species by comparative sequence analysis of the RNA polymerase gene (rpoB). J Clin Microbiol 1999;37:171420.

18. Hwang S, Oh KJ, Jang IH, et al. Evaluation of the diagnostic performance of the advansure TB/NTM real-time PCR kit for detection of mycobacteria. Korean J Clin Microbiol 2011;
14:55-9.

19. Yu JR, Heo ST, Lee KH, et al. Skin and soft tissue infection due to rapidly growing mycobacteria: case series and literature review. Infect Chemother 2013;45:85-93.

20. Dodiuk-Gad R, Dyachenko P, Ziv M, et al. Nontuberculous mycobacterial infections of the skin: a retrospective study of 25 cases. J Am Acad Dermatol 2007;57:413-20.

21. Gillespie $\mathrm{SH}$, Billington O. Activity of moxifloxacin against mycobacteria. J Antimicrob Chemother 1999;44:393-5.

22. Choi GE, Min KN, Won CJ, et al. Activities of moxifloxacin in combination with macrolides against clinical isolates of Mycobacterium abscessus and Mycobacterium massiliense. Antimicrob Agents Chemother 2012;56:3549-55.

23. Reddy P, Das S, Chandler JP, et al. Stenotrophomonas maltophilia meningitis treated with moxifloxacin: a case report and review of the literature. Infect Dis Clin Prac 2006; 14:173-6.

24. Son SW, Kim HJ, Seo JW. A case of Stenotrophomonas maltophilia keratitis effectively treated with moxifloxacin. Korean J Ophthalmol 2011;25:349-51. 